\title{
Utilidad de la plasmaféresis en cuidados intensivos
}

\author{
C. Salazar Ramirez, D. Daga Ruiz*, F. Cota Delgado, C. Fernández Aguirre, J.M. Fernández \\ Añon y J.M. Garcia Fernández
}

Servicio de Medicina Intensiva, Hospital Universitario Virgen de la Victoria, Málaga, España

Recibido el 10 de enero de 2009; aceptado el 30 de marzo de 2009

Disponible en Internet el 17 de octubre de 2009

\section{PALABRAS CLAVE \\ Plasmaféresis; \\ Tratamientos \\ continuos de \\ reemplazo renal; \\ Cuidados intensivos}

\section{KEYWORDS}

Plasmapheresis;

Replacement therapy;

Intensive care

\begin{abstract}
Resumen
Presentamos las plasmaféresis (PMF) realizadas en una unidad de cuidados intensivos (UCI) polivalente de 18 camas en el quinquenio comprendido entre los años 2003-2007. El objetivo del presente artículo es comunicar nuestra experiencia en PMF realizada con monitores específicos para tratamientos continuos de reemplazo renal (TCRR) y evidenciar la versatilidad derivada del uso de estos tratamientos y de estos monitores en las UCl. La utilidad de estos procedimientos abarcan muchos escenarios de la enfermedad crítica ingresada en nuestras unidades (pacientes neurológicos, hematológicos, reumatológicos). En definitiva, nuestra experiencia en PMF nos lleva a concluir que es un tratamiento de depuración extracorpórea sencillo, que puede realizar el personal sanitario de cuidados intensivos en cualquier momento dentro de un amplio espectro de indicaciones clínicas, con monitores de TCRR y con unas complicaciones asociadas a la técnica mínimas y leves. (c) 2009 Elsevier España, S.L. y SEMICYUC. Todos los derechos reservados.
\end{abstract}

\section{Utility of plasmapheresis in intensive care}

\begin{abstract}
We discuss the plasmapheresis (PE) carried out in an 18-bed polyvalent intensive care unit between the years 2003-2007. This article aims to report our experience in plasmapheresis performed with specific monitors for continuous renal replacement therapy (CRRT) that shows the versatility of the use of these procedures in intensive care. The utility of these procedures include many different critical disease settings in our units (neurology, hematology, and rheumatology patients). In short, our experience in PE has led us to the conclusion that plasmapheresis is a simple extracorporeal depuration treatment that can be performed by staff trained in intensive care at any moment within a wide spectrum of clinical indications, with CRRT monitors and with minimum adverse effects.

(c) 2009 Elsevier España, S.L. and SEMICYUC. All rights reserved.
\end{abstract}

*Autor para correspondencia.

Correo electrónico: domingo.daga.sspa@juntadeandalucia.es (D. Daga Ruiz). 


\section{Introducción}

Abel realizó la primera plasmaféresis (PMF) en 1914. En la década de 1970 hay un aumento en el uso de la técnica como tratamiento de muchas enfermedades y es en la década de 1990 cuando se han definido de forma más precisa las indicaciones de la PMF como tratamiento ${ }^{1,2}$.

La PMF se entiende como el procedimiento por el que el plasma se separa de la sangre y se procesa para eliminar selectivamente algunos componentes. Una vez procesado se reinfunde al paciente. El recambio plasmático se define como el procedimiento por el que el plasma se separa de la sangre y lo sustituye un líquido de reposición. En la práctica clínica ambos términos se utilizan como sinónimos, aunque en la inmensa mayoría de las ocasiones se elimina el plasma separado de la sangre total y lo sustituye una solución de reposición isovolumétrica.

Las características del líquido de reposición dependerán del tipo de enfermedad que haya originado el procedimiento.

El mecanismo exacto por el que la PMF tiene un efecto terapéutico se desconoce, aunque se postula que el descenso o eliminación en la concentración de sustancias patológicas del plasma tras realizar el procedimiento induce el efecto terapéutico. Estas sustancias perjudiciales que se eliminan pueden ser anticuerpos, inmunocomplejos, proteínas monoclonales, toxinas, citoquinas-mediadores y otras no conocidas.

Con distintos grados de evidencia, la PMF se ha empleado en el tratamiento de diversas enfermedades, especialmente en las áreas neurológica ${ }^{3}$, hematológica ${ }^{4}$ y reumatológica ${ }^{5}$.

Los métodos de separación del plasma se pueden dividir en:

- centrifugación con flujo discontinuo,

- centrifugación con flujo continuo,

- y filtración.

En el caso de la filtración, la separación del plasma de los componentes celulares de la sangre se realiza mediante un filtro, con una membrana de poro grande $(0,2-0,7 \mu \mathrm{m})$, que extrae moléculas de hasta 3 millones de D. Este plasmafiltro es el elemento central de un circuito extracorpóreo que junto con las tubuladuras y el catéter de acceso venoso será similar a los utilizados en las unidades de cuidados intensivos (UCI) para la realización de otros tratamientos de depuración extracorpórea (TDE) ${ }^{6}$, donde lo que se modificará será el tipo de filtro (tamaño y características de la membrana) y la programación de las diferentes bombas peristálticas, que moverán los distintos líquidos por el circuito extracorpóreo según la terapia depurativa deseada: hemofiltración continua, hemodiafiltración continua, hemodiálisis continua, diálisis con albúmina con sistema MARS ${ }^{\circledR}$ versus Prometheus ${ }^{\circledR}$, plasmafiltración-adsorción continua, etc.

El motivo de la presente nota clínica es exponer la utilidad de la PMF en distintos escenarios de pacientes críticos ingresados en una UCI con amplia experiencia en la utilización de tratamientos continuos de reemplazo renal (TCRR) y con un personal sanitario altamente cualificado en el montaje, cebado, dosificación, monitorización y mante- nimiento de circuitos extracorpóreos las $24 \mathrm{~h}$ del día y los 7 días de la semana.

Presentamos un análisis retrospectivo de una serie de casos llevados a cabo en la UCl del Hospital Virgen de la Victoria de Málaga, un hospital público de tercer nivel que cuenta con 18 camas divididas en 3 áreas, con 6 camas para coronarios y 12 camas para pacientes con problemas médicos o quirúrgicos.

La máquina utilizada para realizar los procedimientos fue el monitor Prisma ${ }^{\mathbb{R}}$ de Hospal S.L. Se insertaron catéteres venosos de doble luz con un diámetro superior a $11 \mathrm{~F}$ por vía femoral. El montaje del sistema se realizó con la técnica habitual de montaje del circuito extracorpóreo y se utilizó un plasmafiltro.

El número y la frecuencia de las sesiones variaron según la enfermedad de base y el curso clínico de la enfermedad. Los líquidos utilizados habitualmente para la reposición variaron en función de la enfermedad de base, y la solución de albúmina al $5 \%$ es la que se usó en la mayoría de las situaciones, o el plasma fresco congelado en el caso de púrpura trombótica trombocitopénica (PTT).

Se incluyeron de forma retrospectiva todos los pacientes que recibieron este procedimiento desde el año 2003 hasta el año 2007 y se analizaron las características basales de los pacientes; las variables analizadas fueron la edad, el sexo, la afección clínica asociada, la indicación ASFA (American Society of Apheresis), los procedimientos asociados (ventilación mecánica, hemofiltración/hemodiálisis, vasopresores), la gravedad de éstos (APACHE II al ingreso y la alta), los efectos adversos durante la técnica y la evolución (tabla 1). Se definieron como efectos adversos asociados al procedimiento las alteraciones hemodinámicas y las complicaciones hemorrágicas o infecciosas; como evolución favorable a la mejoría tanto clínica como de laboratorio, según la enfermedad asociada (para la pancreatitis hiperlipidémica si los triglicéridos alcanzan valores menores de $500 \mathrm{mg} / \mathrm{dl}$; o para el síndrome de Goodpasture cuando los anticuerpos membrana basal fueron negativos); y como evolución desfavorable el fallecimiento del paciente. Las pruebas estadísticas se realizaron según la naturaleza de las variables. Los datos se analizaron con el programa SPSS 11.5.

La población final del estudio constó de 24 pacientes cuyas características basales se muestran en la tabla 1. La edad media de los pacientes ingresados fue de 41,6 \pm 16 años (rango: 18 a 73 años) y predominó el sexo femenino. Se realizaron 64 sesiones (promedio: 2,6; rango: 1 a 7) y la puntuación promedio en la escala de gravedad APACHE II al ingreso fue de $15 \pm 9,7$.

En los resultados obtenidos se observa que las distintas sesiones de plasmaféresis se realizaron sobre una muestra heterogénea de sujetos con enfermedades neuroinmunológicas, reumatológicas, hematológicas y en pancreatitis hiperlipidémicas; y la enfermedad que con más frecuencia originaba el inicio del procedimiento fue la PTT (el 37,5\% de los casos), seguida de pancreatitis hiperlipidémica (25\%) - figura 1-; un 16,7\% de las plasmaféresis correspondieron a pacientes con vasculitis y síndrome renal-pulmonar, y el resto de los pacientes que recibieron plasmaféresis correspondieron a cuadros neuroinmunológicos como la miastenia gravis $(8,3 \%)$ y síndrome de Guillain-Barré $(8,3 \%)$ (tabla 2). 


\begin{tabular}{|c|c|c|c|c|c|c|c|c|c|}
\hline Paciente & Edad & Sexo & Enfermedad previa & Indicación PE & $\begin{array}{l}\text { Indicación } \\
\text { ASFA }\end{array}$ & $\begin{array}{l}\text { Procedimientos } \\
\text { asociados }\end{array}$ & $\begin{array}{l}\text { APACHE II al } \\
\text { ingreso }\end{array}$ & $\begin{array}{l}\text { APACHE II al } \\
\text { alta }\end{array}$ & Evolución \\
\hline 1 & 52 & Fem & Miastenia gravis & Miastenia gravis & I & VM & 13 & 11 & Fav \\
\hline 2 & 72 & Fem & ACV previo & $\mathrm{SHU} / \mathrm{PTT}$ & I & VM, VS & 26 & 18 & Fav \\
\hline 3 & 33 & Mas & No & SHU/PTT & I & No & 10 & 10 & Fav \\
\hline 4 & 35 & Fem & LES & GNRP & II & VM, VS, TDE & 28 & 31 & Des \\
\hline 5 & 22 & Fem & No & Sd. Riñón-pulmón & II & VM, VS, TDE & 22 & 16 & Fav \\
\hline 6 & 68 & Mas & ACV previo & SHU/PTT & 1 & VM, VS & 19 & 19 & Des \\
\hline 7 & 18 & Fem & No & Sd. Riñón-pulmón & I & VM, VS, TDE & 19 & 16 & Fav \\
\hline 8 & 45 & Fem & HTA & $\mathrm{SHU} / \mathrm{PTT}$ & I & No & 6 & 4 & Fav \\
\hline 9 & 28 & Fem & No & SHU/PTT & I & VM & 5 & 4 & Fav \\
\hline 10 & 39 & Fem & PTT & $\mathrm{SHU} / \mathrm{PTT}$ & 1 & No & 7 & 5 & Fav \\
\hline 11 & 42 & Mas & $\begin{array}{l}\text { Vasculitis de } \\
\text { Wegener }\end{array}$ & Sd. Riñón-pulmón & II & VM, VS, TDE & 32 & 22 & Fav \\
\hline 12 & 36 & Fem & $\begin{array}{l}\text { Sd. } \\
\text { Quilomicronémico }\end{array}$ & $\begin{array}{l}\text { Pancreatitis } \\
\text { hiperlipidémica }\end{array}$ & I & No & 4 & 4 & Fav \\
\hline 13 & 20 & Fem & No & SHU/PTT & I & $\mathrm{HF}$ & 6 & 5 & Fav \\
\hline 14 & 47 & Fem & Miastenia gravis & Miastenia gravis & 1 & VM, VS, TDE & 26 & 28 & Des \\
\hline 15 & 27 & Fem & Hipertrigliceridemia & $\begin{array}{l}\text { Pancreatitis } \\
\text { hiperlipidémica }\end{array}$ & 1 & No & 15 & 10 & Fav \\
\hline 16 & 29 & Mas & Hipertrigliceridemia & $\begin{array}{l}\text { pancreatitis } \\
\text { hiperlipidémica }\end{array}$ & I & No & 8 & 7 & Fav \\
\hline 17 & 69 & Mas & Vasculitis & Sd. Riñón-pulmón & II & VM, VS, TDE & 36 & 36 & Des \\
\hline 18 & 73 & Fem & No & Sd. Guillain-Barré & 1 & VM & 12 & 10 & Fav \\
\hline 19 & 48 & Mas & Hipertrigliceridemia & $\begin{array}{l}\text { Pancreatitis } \\
\text { hiperlipidémica }\end{array}$ & 1 & VM, VS, TDE & 18 & 26 & Des \\
\hline 20 & 44 & Mas & Hipertrigliceridemia & $\begin{array}{l}\text { Pancreatitis } \\
\text { hiperlipidémica }\end{array}$ & I & VM, VS, TDE & 26 & 30 & Des \\
\hline 21 & 47 & Fem & Hipertrigliceridemia & $\begin{array}{l}\text { Pancreatitis } \\
\text { hiperlipidémica }\end{array}$ & I & No & 6 & 5 & Fav \\
\hline 22 & 29 & Mas & No & SHU/PTT & 1 & No & 15 & 8 & Fav \\
\hline 23 & 53 & Mas & No & SHU/PTT & I & No & 4 & 4 & Fav \\
\hline 24 & 24 & Fem & No & Sd. Guillain-Barré & I & VM & 6 & 5 & Fav \\
\hline
\end{tabular}




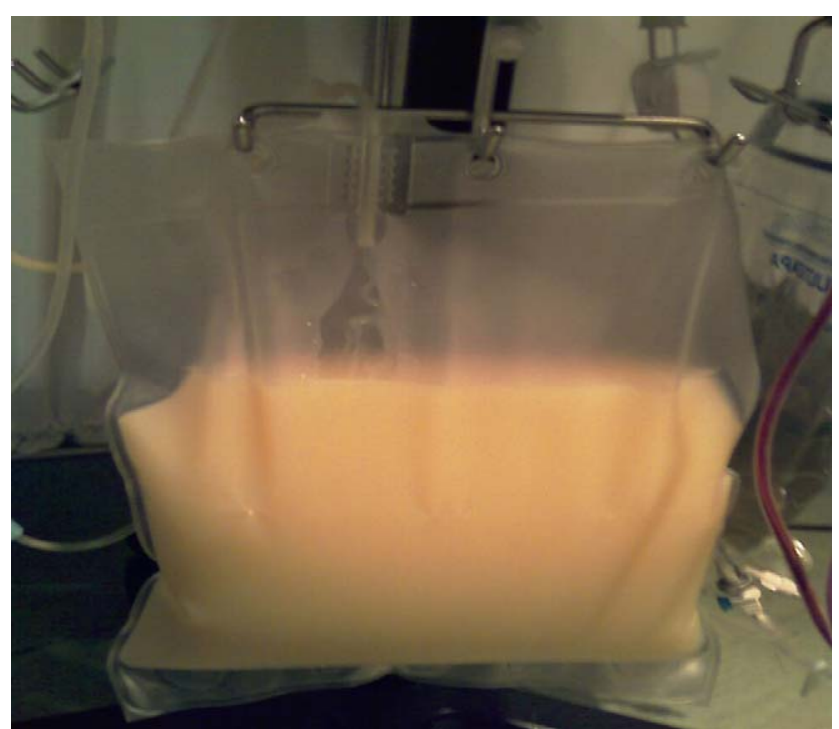

Figura 1 Bolsa de efluente de aspecto lechoso. Pancreatitis hiperlipidémica.

Tabla 2 Indicaciones para la realización de plasmaféresis

\begin{tabular}{llr}
\hline Enfermedad & Número de casos & $\%$ \\
\hline PTT/SHU & 9 & 37 \\
Pancreatitis & 6 & 25 \\
Vasculitis vaso pequeño & 4 & 16 \\
Miastenia gravis & 2 & 8 \\
Guillain-Barré & 2 & 8 \\
GNRP & 1 & 4 \\
\hline
\end{tabular}

GNRP: glomerulonefritis rápidamente progresiva; PTT: púrpura trombótica trombocitopénica; SHU: síndrome hemolítico urémico.

Los pacientes que recibieron estos procedimientos presentaban una situación clínica grave y de alta complejidad, que se observó por la alta incidencia de tratamientos de soporte simultáneos que recibía el $62,5 \%$ de nuestros pacientes, tales como ventilación mecánica, drogas vasoactivas y otros TDE de soporte renal.

En nuestra muestra constatamos una disminución de los valores promedios de los índices de gravedad y disfunción de órganos (determinados por el uso de APACHE II al ingreso y al alta -15 versus $13,9-$, así como la necesidad de soporte orgánico) tras las sesiones de plasmaféresis administradas.

En relación con el número de complicaciones, todas fueron mínimas y no se presentaron las comúnmente descritas en la literatura médica ${ }^{7-9}$. Observamos episodios de hipotensión transitorios en el $30 \%$ de los procedimientos, que respondieron con cargas de volumen sin necesidad de suspender el tratamiento de aféresis. No registramos ningún episodio de hipocalcemia sintomática, ni hemorragia atribuibles al deterioro de pruebas de coagulación ni ningún episodio de infección relacionado con el catéter; todo esto coloca a la plasmaféresis como un procedimiento seguro.

La evolución fue favorable en la mayoría de nuestros pacientes y un $25 \%$ tuvo una evolución calificada como desfavorable o tórpida (fallecimiento). Ninguna muerte fue atribuible al procedimiento, sino más bien debido a la gravedad de la enfermedad de base y sus complicaciones.

\section{Discusión}

Los tratamientos de reemplazo renal extracorpóreos se iniciaron a mediados del siglo xx con la hemodiálisis intermitente en el seno del fracaso renal crónico en fase terminal.

No es hasta la década de 1970 cuando se describe la hemofiltración arteriovenosa continua como una técnica de soporte renal para pacientes críticos en fracaso renal agudo (FRA). Desde entonces, los TCRR han ido creciendo de forma exponencial como una técnica de soporte renal en el seno de la disfunción renal del paciente crítico ingresado en $\mathrm{UCl}$, de forma que en nuestros días no es fácil imaginar una $\mathrm{UCl}$ sin monitores específicos para la realización de los TCRR.

En el estudio que realizó el grupo de trabajo de Cuidados Intensivos Nefrológicos de la SEMICYUC, en el que participaron 41 servicios de Medicina Intensiva del país, el 38\% del FRA recibió reemplazo renal extracorpóreo. De éstos, el $84 \%$ fueron TCRR.

En el escenario antes descrito, ya desde la década de 1980 hubo autores que empezaron a utilizar los TDE en el contexto del paciente crítico sin FRA, o sin que éste fuera la única indicación para iniciarlos.

El presente artículo presenta nuestra experiencia en PMF mediante la utilización de monitores específicos para TCRR y demuestra la versatilidad que se deriva del uso de éstos monitores en las $\mathrm{UCl}$.

Una vez aceptada la técnica/tratamiento extracorpóreo por el personal sanitario de la unidad, tanto médicos como enfermería, la utilidad de estos procedimientos puede abarcar muchos escenarios de la enfermedad crítica ingresada en nuestras unidades y ampliar significativamente nuestra cartera de servicios.

En definitiva, nuestra experiencia en PMF en estos últimos 6 años nos lleva a concluir que:

1. Es una técnica/tratamiento extracorpóreo sencillo y de fácil realización.

2. Puede realizarse tanto de forma urgente como programada en una $\mathrm{UCI}$ con una amplia experiencia en la realización de TCRR, a cualquier hora del día y en cualquier día de la semana, sin contar con más personal sanitario que el específico de cuidados intensivos.

3. El espectro de indicaciones clínicas que podemos abarcar incluye enfermedades diversas tanto en su etiopatogenia como en su pronóstico y evolución. En nuestra serie tuvimos pacientes inmunoneurológicos, hematológicos, reumatológicos y una serie de 6 casos de pancreatitis hiperlipidémicas.

4. En nuestra experiencia, las complicaciones derivadas de la técnica fueron mínimas y en todas las ocasiones leves.

\section{Bibliografía}

1. Rososhansky S, Szymansky I. Cinical applications of therapeutic hemapheresis. J Intensive Care Med. 1996;3:149-61.

2. Clark WF, Rock G, Buskard N, Shumak KH, LeBlond P, Anderson D, et al. Terapeutic plasma exchange: An update from the Canadian Apheresis Group. Ann Intern Med. 1999;131:453-62. 
3. Ciavarella D, Wuest D, Strauss RG, Gilcher R, Kasprisin D, Kiprov $D$, et al. Cinical applications of therapeutic apheresis: Management of neurologic disorders. J Clin Apheresis. 1993;8:242-57.

4. Byrnes JJ, Khurana M. Teatment of thrombotic thrombocytopenic purpura with plasmapheresis. N Engl J Med. 1977;297:1386-9.

5. Wallace DJ. Apheresis for lupus erythematosus: State of the art. Lupus. 2001;10:193-6.

6. Herrera Gutiérrez ME, Daga Ruiz D, Seller Pérez G, García Alcántara A, De la Rubia de Gracia C, Ruiz del Fresno L. Uso de las técnicas continuas de reemplazo renal en las Unidades de
Cuidados Intensivos en España. Una encuesta a nivel nacional. Med Intensiva. 2000;24:341-7.

7. Benítez C, Andresen M. Indications, adverse effects and results of plasmapheresis in critical care patients. Rev Méd Chile. 2005;133:1441-8.

8. Morkrzycki MH, Kaplan AA. Therapeutic plasma exchange: Complications and management. Am J Kidney Dis. 1994;23: 817-827.

9. Reimann PM, Mason PD. Plasmaféresis: técnica y complicaciones. Intensive Care Med. 1990;16:3-10. 